\title{
A Comprehensive Assessment on IOT Devices with Data Mining Techniques
}

\author{
G. Vijay Kumar a, 1 , M. Sreedevi a , Arvind Yadav a, B. Aruna ${ }^{\text {a }}$ \\ ${ }^{a}$ Department of Computer Science and Engineering, Koneru Lakshmaiah \\ Education Foundation, Greenfields, Vaddeswaram, Guntur-522502, India
}

\begin{abstract}
Now at present development the entire world using vast variety of smart devices associated among sensors \& handful of actuators. There is an enormous progress within the field of electronic communication; processing the data through devices and the bandwidth in internet technologies makes very easy to access and to interact with the variety of devices all over the whole world. There is a wide range research in the area of Internet of Things (IoT) along Cloud Technologies making to build incredible data which are creating from this type of heterogeneous environments and can be able to transform into a valuable knowledge with the help of data mining techniques. The knowledge that is generated will takes a crucial role in making intellectual decisions and also be a best possible resource management and services. In this paper we organized a comprehensive assessment on various data mining techniques engaged with small and large scale IoT applications to make the environment smart.
\end{abstract}

Keywords. Internet of Things, Sensors, Actuators, Cloud, Data Mining.

\section{Introduction}

At the present time all kinds of Industries are utilizing and contributing their work and doing Examination on the gadgets which we use daily and throughout the world in the IoT technology. The companies like Cisco, Microsoft, Google, and Research societies together with Internet of Things (IoT), Wireless Sensor Networks (WSN), Mobile Computing (MC), Cyber-Physical System (CPS) etc., the entire world functioning and investigate to contribute their work and curiosity to make the urban areas as Smart Environment [1-3]. In IoT, the things which we can likewise call as the Objects are its availability with Internet. We can say that the web is additionally utilized as mechanism of network and furthermore be utilized as force to run gadgets. For any type of devices, the correspondence plays a essential task in the network. The devices retrieve the information and need to share this information to different gadgets and the server farm needs a medium to do this where web will assist the information with being shared between different gadgets and the server farm. For this the gadgets ought to have a one-of-a-kind personality to recognize it in organization, it should be distinguished naturally, and ought to impart and impart the information to one another and the people. They should settle on choice without help from anyone else or follow the human orders [4].

\footnotetext{
${ }^{1}$ G. Vijay Kumar,Department of Computer Science and Engineering, Koneru Lakshmaiah Education Foundation, Greenfields, Vaddeswaram, Email: gvijay_73@kluniversity.in.
} 
Presently the inquiry emerges that why would that be a great deal of examination going on IoT innovation and Industries are moving towards IoT? The research which is going on shows that urban areas will be overlaid among detecting and activation gadgets in the following 10 Years. The sensors and actuators will increment soon after quickly detecting and activating gadgets will expand threefold as much as populace of individuals. Ericsson and Cisco anticipated that "50 billion of little inserted actuators and sensors associated with the web before 2020 year with Internet of Things will make 14.4 trillion dollars of significant worth in question for businesses in the following decade" [5]. So, we can say that, a huge number of connected heterogeneous devices will form IoT environment [6-9]. The detecting gadgets which are in IoT climate produces huge amount of data. This data which is sensed, initially called as Raw data. This information is at first not introduced in Human justifiable language and should be handled. The information may have a place with a framework or any kind of utilization in the Smart IoT climate. The foundation may comprise of gadgets information just as the organization information or any sort of information system in the IoT climate. The information tangles situate with a little sort of utilization or huge kind of use. The information should be changed over into human reasonable language where Data Mining procedures will assist us with recovering the significant data from the information [10]. The installed gadgets in IoT climate may create colossal information might be to serve various applications. Here a unified middleware to improvement cycle simpler, give protection, security inside assorted applications and administrations [11].

\section{Related Work}

In paper [4] they have reviewed study on data mining technologies with Internet of Things and this paper mainly focused on Application of data mining techniques over Internet of Things and this reference studied on IOT data problems be able to solve through "data mining Technologies like Clustering, Classification and frequent pattern mining for IOT" [4]. In paper [12] "they have assessed IoT and big data analytics. Big data analytics be a emerging technology is a key to IoT for decision making". In paper [13] "they made a heart-beat monitoring system using data mining algorithms like classification" [14], clustering [15], "and frequent/sequential pattern mining" [16,17] "from IoT applications". They also represented "an overview of cloud assisted system architecture and Data mining process".

In reference [18] a mining framework that is able to professionally and confidentially recognize the measures into IoT application. In paper [19] they proposed a model in requisites of accuracy or optimization. Some researchers focused on this model on real time applications. In paper [20] they have a detailed study on data mining applications and 3 different views they are knowledge view, technique view, and application view and data mining technologies are integrated with IoT Technologies for better system optimization and accuracy. 


\section{Key Data Mining Methodologies}

A tremendous measure of information is information is available in the climate around us. It appears to be unusable to make this climate strongly having without suitably using the information mining advancements. Information mining can be a main learning with computerization today. Figure 1 the PC helped learning develops all the more accurately when acted in numerous layers in a progressive way.

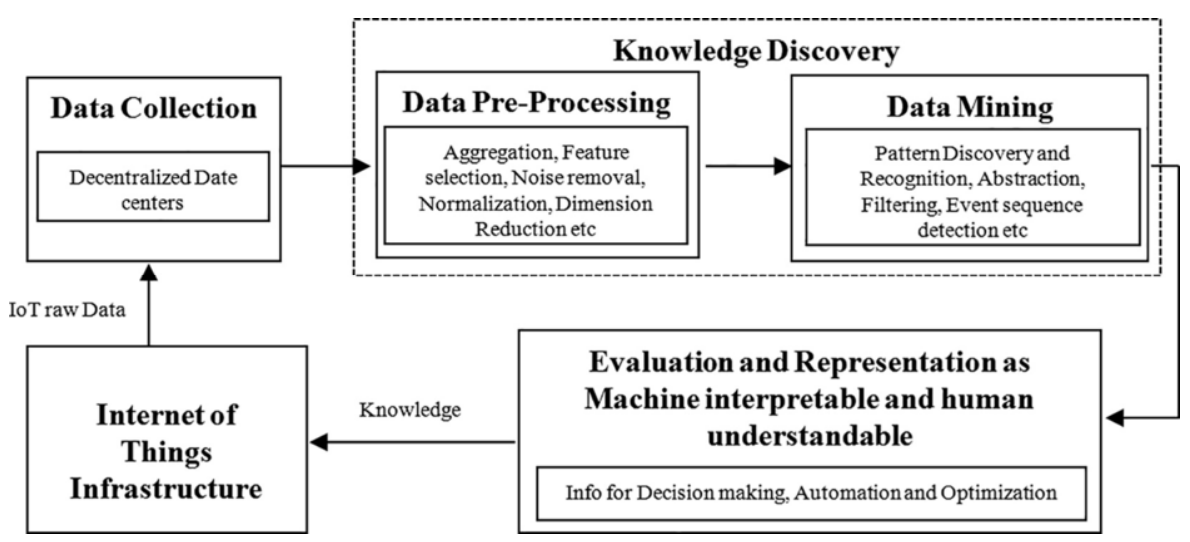

Figure 1. Process of Data Mining in the environment of Internet of Things

\subsection{Classification}

Characterization is an information mining capacity that allots things in an assortment to target classifications or classes. The objective of characterization is to precisely foresee the objective group in the information for each case. For instance, a characterization model can be consumed to recognize advance candidates as low, medium, or high credit hazards. This assessment is used to recuperate huge and significant information about data, and metadata. This data mining procedure helps with gathering data in different classes.

An order task begins with an informational index in which the class tasks are known. On behalf of instance, an order representation that predicts credit danger might be produced dependent on noticed information for some candidates throughout some extend of time. Notwithstanding the chronicled FICO score of the information may track employment history, number and sort of ventures, home proprietorship or rental, extended periods of living arrangement, etc. The FICO assessment will be the objective for different characteristics and the indicators, and the information for every client would comprise a case. 


\subsection{Clustering}

In clustering or assembling of various objects are delegate comparable items. Informational indexes are isolated into various clusters for examination which depends on the likeness of the information. Later the collection of information into a variety of clusters, a mark is doled out to the cluster causes in regulating to the development by grouping. Bunch investigation in Data Mining Figure 2 involve in discovering the gathering of articles which be like each other in the gathering however are not the same as the item in different gatherings.

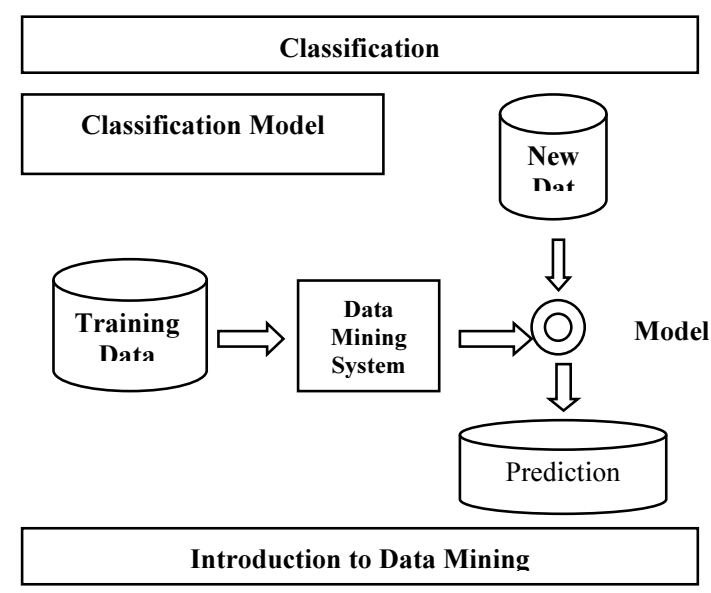

Figure 2. Classification Model

Data Mining cluster analysis applications:

- It helps in distributing records on the web for information revelation.

- It be able to utilized to decide plant and creature scientific classifications, arrangement of qualities with similar functionalities and understanding the structure innate to populaces.

- It helps the ID territories of comparative land to utilize on ground perception and ID of house bunches as per house type, esteem, and topographical area.

\subsection{Use of Clustering}

Scalability: Adaptability in bunching suggests that as we support the measure of information protests, figure 3 an opportunity to perform grouping ought to around scale to the multifaceted nature request of the calculation. 

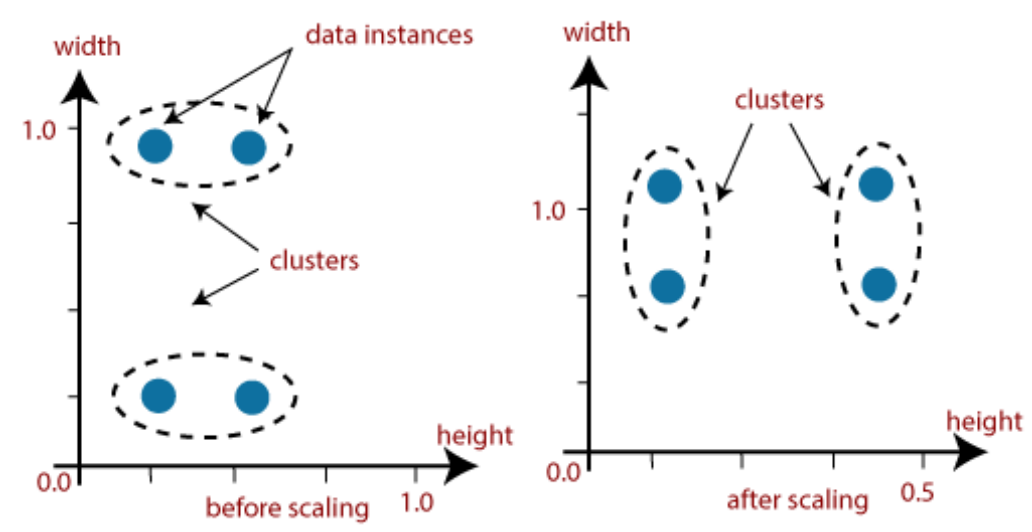

Figure 3. Showing example where Scalability may leads to wrong result

Interpretability: The outcomes of clustering should be interpretable, comprehensible, and usable.

In View of Smart Agriculture: In horticulture creation, the amount and quality necessities of future food and sustenance supplements are significant in our everyday life. As the days are passing the ascent in populace builds step by step. Along these lines, utilizing the idea IOT (internet of things) we can build up this farming framework with huge numbers of the forthcoming cutting-edge innovations. The primary attributes like organization availability, low force sensors and actuators arrangement are the fundamental significant item to be utilized in this venture. For making this agribusiness framework here we use IoT with distributed computing for the better process. The calculation utilized here is the enormous information mining to get the arrangement all the simpler for enhancing and improving shrewd agribusiness framework. Presently in these current days there are a considerable lot of the unexperienced of food should me more and acceptable quality. By the new innovations like completely robotized machines are to be utilized in the horticulture to plant the seeds without the assistance of numerous ranchers. Dribble water system, water reusing and the composts can likewise spread to the plants effectively with these mechanized machines. Getting data (moisture, temperature) from sensor hubs like zigbee sensors and after to lessen the clamor in the detected information play the pressure utilizing the expectation with sifting and afterward the exchange of the information utilizing IoT door and a solid shape introduced on raspberry pi. Expectation about the harvest development utilizing the calculation as choice tree. The keen articles like submerged sensors and AUVs (for boor), Burried sensors (for loUGT), Nano-satellites (for lost). The correspondences, systems administration and confinement of the brilliant submerged investigation and observing, expectation farming, selsmic investigation , and checking of oil and gas fields, worldwide network past earth, far off detecting and situating. They will be the significant uses of X-IoT.

In View of Smart Transportation: Within urban communities will have a substantial traffic because of the expanding of populace step by step. Along these lines, because of this substantial traffic, numerous mishaps are looking with this traffic. With the idea of the IOT (Internet of Things) we can make the improvement in this transportation to 
lessen mishaps. Utilizing the sensors in the vehicle to advise us that to wear safety belt, and when a driver crosses the speed of $80 \mathrm{kmph}$ these sensors will be frightened so the driver can diminish speed. To make framework for vehicle aware of try not to glitch condition. For keeping up distance between the vehicle to vehicle is likewise very security insurance to the explorers. The information as well as trade a few administrations information Like security, solace and productivity for social great.

\section{Conclusion}

This paper demonstrated a systematic and specified evaluate of Data mining algorithms like classification and clustering mining from IoT applications perspective. It is reviewed and organized in tabular issues. We descriptively analyzed the functions like Smart Home, Smart Healthcare, Smart Grid, Ambient Assistant Living, Smart Manufacturing, Smart Agriculture, Industrial IoT, and transportation taking place the establishment of records mining applied sciences employed towards the information translation. Here the conversion will increase more complex interested in today's big information to create IoT environment. In addition, we demonstrated the gadget structure in second section and Data mining manner in the above sections for IoT to pre-processing as well as understanding discovery performs in necessary position amongst the layers. Structures that consist of a range of diverse clever devices and with the purpose of creating heterogeneous data for pre-processing top-notch importance. The machine boosts knowledge discovery and overall presentation with the aid of extra splendid and superior carrier suggestions.

\section{References}

[1] Stankovic, John., "Research directions for the internet of things", IEEE Internet Things Journal, 1 (1): pp. 3-9, 2014.

[2] Kravchenko Yurii, Olena starkova. et al., "Technology Analysis for smart Home Implementation", In proceedings of 4th International Scientific-Practical Conference on Problems of Infocommunications, Science and Technology, Ukraine, pp.579-584, Oct 2017.

[3] Miorandi Daniele., De Pellegrini, F., et al., "Internet of things: vision, applications and research challenges", Elsevier Journal. Ad Hoc Netw. 10 (7), pp.1497-1516, 2012.

[4] Tsai Chun.W., et al., "Data Mining for Internet of Things - A Survey", IEEE Communication Surveys and Tutorials. Vol. 16 issue 1, pp. 451, 2014.

[5] Google 2017,Big data online Available: https://cloud.google.com/whatis-big-data/.

[6] Uusitola Mikko A., "Global vision for the future wireless world from the WWRF", IEEE Vehicular Technology Magazine, vol. 1 issue 2, pp. 4-8, 2006.

[7] Ortiz Antonio M., Hussein Dina., et al., The cluster between internet of things and social networks: review and research challenges. IEEE Internet of Things Journal, vol. 1 issue 3, pp. 206-215, 2014.

[8] Bijarbooneh Farshid Hassani., Du Wei., et.al, "Cloud-Assisted Data Fusion and Sensor Selection for Internet of Things", IEEE Internet of Things Journal, vol. 3 issue 3, pp.257-268, June 2016.

[9] Potharaju, S. P., \& Sreedevi, M. (2018). A novel cluster of quarter feature selection based on symmetrical uncertainty. Gazi University Journal of Science, 31(2), 456-470.

[10] Mohammad Abdul Razzaque, Marija Milojevic-Jevric et al., "Middleware for internet of things-a survey", IEEE Internet Things Journal, vol 3 issue 1, pages 70-95, Feb 2016.

[11] Mohsen Marjani, Fariza Nasaruddin et al., "Big IoT Data Analytics: Architecture, Opportunities, and Open Research Challenges" IEEE Access, vol 5, pp, 5247-5261, 2017.

[12] Vijay Kumar G, Bharadwaja A, et al., "Temperature and heart beat monitoring system using IOT", Proceedings of ICEI 2017- 2018, pp. 692-695, Jan 2018. 
[13] Vijay Kumar G, Sreedevi M., et al., "Incremental mining of popular patterns from transactional databases", IJET, vol 7 issue 2.7, pp. 636-641, 2018

[14] Vijay Kumar G, Sreedevi M et al., "Regular frequent crime pattern mining on crime datasets" IJET, vol 7 issue 2.7, pp. 972-975, 2018.

[15] Vijay Kumar G, Krishna Chaitanya T, et al., "Mining Popular Patterns from Multidimensional Database", Indian Journal of Science and Technology, Vol 9 issue 17, DOI: 10.17485/ijst/2016/v9i17/93106, May 2016.

[16] Vijay Kumar G, and ValliKumari. V, "Incremental mining for regular frequent patterns in vertical format”, IJET, Volume 5, Issue 2, pp. 1506-1511, 2013.

[17] Samer Samarah, Mohammed Gh. Al Zamil et al., "An Efficient Activity Recognition Framework: Toward Privacy-Sensitive Health Data Sensing”, IEEE Access, vol 5, pp. 3848-3859, 2017.

[18] Zainal Abedin, Prashengit Dhar et al., "Traffic Sign Detection and Recognition Using Fuzzy Segmentation Approach and Artificial Neural Network Classifier Respectively", International Conference on Electrical, Computer and Communication Engineering (ECCE), pp. 518-523, 2017.

[19] Vengatesan K., Kumar A., Parthibhan M., Singhal A., Rajesh R. (2020) Analysis of Mirai Botnet Malware Issues and Its Prediction Methods in Internet of Things. In: Pandian A., Senjyu T., Islam S., Wang H. (eds) Proceeding of the International Conference on Computer Networks, Big Data and IoT (ICCBI - 2018). ICCBI 2018. Lecture Notes on Data Engineering and Communications Technologies, vol 31.

[20] Feng Chen, Pan Deng et al., "Data Mining for the Internet of Things: Literature Review and Challenges", International Journal of Distributed Sensor Networks, vol. 11 issue 8, 2015. 